\title{
Bayesian Estimations under the Weighted LINEX Loss Function Based on Upper Record Values
}

\author{
Fuad S. Al-Duais $\mathbb{i D}^{1,2}$ \\ ${ }^{1}$ Mathematics Department, College of Humanities and Science in Al Aflaj, Prince Sattam Bin Abdulaziz University, \\ Al Aflaj, Saudi Arabia \\ ${ }^{2}$ Administration Department, Administrative Science College, Thamar University, Thamar, Yemen \\ Correspondence should be addressed to Fuad S. Al-Duais; f.alduais@psau.edu.sa
}

Received 15 March 2021; Revised 11 April 2021; Accepted 15 April 2021; Published 26 April 2021

Academic Editor: Ahmed Mostafa Khalil

Copyright (C) 2021 Fuad S. Al-Duais. This is an open access article distributed under the Creative Commons Attribution License, which permits unrestricted use, distribution, and reproduction in any medium, provided the original work is properly cited.

\begin{abstract}
The essential objective of this research is to develop a linear exponential (LINEX) loss function to estimate the parameters and reliability function of the Weibull distribution (WD) based on upper record values when both shape and scale parameters are unknown. We perform this by merging a weight into LINEX to produce a new loss function called the weighted linear exponential (WLINEX) loss function. Then, we utilized WLINEX to derive the parameters and reliability function of the WD. Next, we compared the performance of the proposed method (WLINEX) in this work with Bayesian estimation using the LINEX loss function, Bayesian estimation using the squared-error (SEL) loss function, and maximum likelihood estimation (MLE). The evaluation depended on the difference between the estimated parameters and the parameters of completed data. The results revealed that the proposed method is the best for estimating parameters and has good performance for estimating reliability.
\end{abstract}

\section{Introduction}

Record values appeared naturally in many real-life applications such as data relating to sport, weather, and lifetesting studies. Many authors studied record values and the associated statistics such as [1-6]. Furthermore, there are several studies which discussed some inferential methods based on record values for the Rayleigh [7, 8], Weibull [9], inverse Weibull [10, 11], exponentiated Family [12, 13], Lomax [14, 15], power Lindley model [16], exponentiated Weibull $[17,18]$, and normal distribution [19].

In this paper, we derive the Bayes estimator under the weighted linear exponential (WLINEX) loss function to obtain parameters and the reliability function of the Weibull distribution based on record values. Afterwards, we compare the proposed model with others.

Let $X_{1}, X_{2}, X_{3}, \ldots$ be a sequence of independent and identically distributed (iid) random variables with (c.d.f) $F(x)$ and (p.d.f) $f(x)$. Set $Y_{m}=\max \left(X_{1}, X_{2}, X_{3}, \ldots, X_{m}\right)$, $m \geq 1$, and we say that $X_{j}$ is an upper record and denoted by $X_{U(i)}$ if $Y_{j}>Y_{j-1}, j>1$.
Assume that $X_{U(1)}, X_{U(2)}, X_{U(3)}, \ldots, X_{U(m)}$ are the first $m$-upper record values arising from a sequence $\left\{X_{i}\right\}$ of (iid) Weibull variables with probability density function (p.d.f)

$$
f(x ; \alpha, \beta)= \begin{cases}\alpha \beta x^{\alpha-1} \exp \left[-\beta x^{\alpha}\right], & x \geq 0, \alpha, \beta>0, \\ 0, & \text { o.w }\end{cases}
$$

and cumulative distribution function (c.d.f)

$$
F(x ; \alpha, \beta)=1-\exp \left[-\beta x^{\alpha}\right], \quad x \geq 0, \alpha, \beta>0,
$$

where $\beta$ and $\alpha$ are scale and shape parameters, respectively. Additionally, the reliability function $R(t)$ and the hazard (instantaneous failure rate) function $H(t)$ at mission time $t$ for the Weibull distribution are given by

$$
\begin{aligned}
R(t ; \alpha, \beta) & =1-F_{T}(t ; \alpha, \beta)=\exp \left[-\beta t^{\alpha}\right], \quad t \geq 0, \\
H(t) & =\alpha \beta t^{\alpha-1}, \quad t \geq 0 .
\end{aligned}
$$




\section{Maximum Likelihood Estimates (MLEs)}

In this section, we discuss the maximum likelihood estimates of the parameters of WD given in (1) when the available data are record values.

Suppose we observe the first $m$-upper record values, each of which has the Weibull distribution whose p.d.f and c.d.f are given by (1) and (2), respectively. Based on these upper record values, for simplicity of notation, we will use $x_{i}$ instead of $X_{U(i)}$. We have the joint density function of the first $m$-upper record values $x \equiv x_{U(1)}, x_{U(2)}, x_{U(3)}, \ldots, x_{U(m)}$ which is given according to Arnold et al. [3] by

$$
\begin{aligned}
f_{1,2,3, \ldots, m}\left(X_{U(1)}, X_{U(2)}, X_{U(3)}, \ldots, X_{U(m)}\right) & =f\left(x_{U(m)}\right) \prod_{i=1}^{m-1} \frac{f\left(x_{U(i)}\right)}{1-F\left(x_{U(i)}\right)}, \\
0 & \leq X_{U(1)}<X_{U(2)}<X_{U(3)}, \ldots,<X_{U(m)}<\infty
\end{aligned}
$$

where $f(\cdot)$, and $F(\cdot)$ are given, by (1) and (2), respectively, after replacing $x$ by $x_{U(i)}$.

The likelihood function based on the $m$-upper record values $x$ is given as follows:

$$
\ell\left(\alpha, \frac{\beta}{x}\right)=(\alpha \beta)^{m} u \exp \left[-\beta T_{m}\right], \quad u=\prod_{i=1}^{m} x_{U(i)}^{\alpha-1}, T_{m}=x_{U(m)}^{\alpha} .
$$

From (6), the natural logarithm of the likelihood function is given by

$$
L(\alpha, \beta \mid x) \equiv \operatorname{Ln}(\ell)=m \operatorname{Ln}(\alpha \beta)-\beta T_{m}+(\alpha-1) \sum_{i=1}^{m} \operatorname{Ln}\left(x_{U(i)}\right),
$$

where $T_{m}$ is given by equation (6).

When both $\alpha$ and $\beta$ are unknown, the maximum likelihood estimates of $\widehat{\beta}_{\mathrm{ML}}$ and $\widehat{\alpha}_{\mathrm{ML}}$ can be obtained from (7) by solving the following two equations:

$$
\begin{gathered}
\widehat{\alpha}_{\mathrm{ML}}=\frac{m}{m \operatorname{Ln}\left(x_{U(m)}\right)-\sum_{i=1}^{m} \operatorname{Ln}\left(x_{U(i)}\right)}, \\
\widehat{\beta}_{\mathrm{ML}}=\frac{m}{\widehat{x}_{U(m)}} .
\end{gathered}
$$

By the invariance property of the MLE, reliability function $-\widehat{R}(t)_{\mathrm{ML}}$ of $R(t)$ can be obtained as given by (3) after replacing $\widehat{\alpha}_{\mathrm{ML}}$ and $\widehat{\beta}_{\mathrm{ML}}$ by:

$$
\widehat{R}(t)_{\mathrm{ML}}=\exp \left[-\widehat{\beta}_{\mathrm{ML}} \widehat{\alpha}^{\widehat{\alpha}_{\mathrm{ML}}}\right], \quad t \geq 0
$$

\section{Loss Function}

We consider three different loss functions.

\subsection{Squared-Error Loss Function (SEL)}

$$
L(\widehat{\theta}, \theta)=(\widehat{\theta}-\theta)^{2}
$$

The squared-error loss function (quadratic loss) is classified as a symmetric function and associates equal importance to the losses for overestimation and underestimation of equal magnitude. The Bayes estimate $\left(\widehat{\theta}_{\mathrm{BS}}\right)$ under the above loss function is the posterior mean, i.e., $\widehat{\theta}_{\mathrm{Bs}}=E(\theta)$.

3.2. LINEX Loss Function. The LINEX loss function for $\theta$ can be expressed as [20]

$$
L(\Delta) \propto \exp [a \Delta]-a \Delta-1, \quad a \neq 0,
$$

where $\Delta=(\widehat{\theta}-\theta)$. Here, $a$ represents the shape parameter of the loss function. The behavior of the LINEX loss function changes with the choice of $a$. In particular, if $a$ is close to zero, this loss function is close to the SEL loss function and therefore almost symmetric.

The Bayes estimator of $\theta$ denoted by $\widehat{\theta}_{\mathrm{BL}}$, depending on the LINEX loss function, is given by

$$
\widehat{\theta}_{\mathrm{BL}}=-\frac{1}{a} \ln \left[\mathrm{E}_{\theta}(\exp \{-a \theta\})\right], \quad a \neq 0,
$$

provided that $E_{\theta}=\left(e^{-a \theta}\right)$ exists and is finite, where $E_{\theta}$ denotes the expected value.

3.3. Weighted LINEX Loss Function (WLINEX). This function is proposed by the researcher depending on the weighted LINEX loss function (WLINEX) as follows:

$$
L_{w}(\widehat{\theta}-\theta)=w(\theta)[\exp [a \Delta]-a \Delta-1], \quad a \neq 0,
$$

where $\widehat{\theta}$ represents the estimated parameter that makes the expectation of the loss function (equation (14)) as small as possible. The value $w(\theta)$ represents the proposed weighted function, which is given as follows:

$$
w(\theta)=\exp [-z \theta]
$$

Depending on the posterior distribution of parameter $\theta$ and by using the proposed weighted function, we get the Bayesian estimation of parameter $\theta$ as follows: 


$$
\begin{aligned}
\mathrm{E} L_{w}(\widehat{\theta}, \theta)= & \int_{\forall \theta} L_{w}(\widehat{\theta}, \theta) f(\theta \mid x) \mathrm{d} \theta \\
= & \int_{\forall \theta} w(\theta)[\exp [a(\widehat{\theta}-\theta)]-a(\widehat{\theta}-\theta)-1] f(\theta \mid x) \mathrm{d} \theta \\
= & \int_{\forall \theta} \exp [-z \theta] \exp [a(\widehat{\theta}-\theta)] f(\theta \mid x) \mathrm{d} \theta-\int_{\forall \theta} \exp [-z \theta]\{a(\widehat{\theta}-\theta) f(\theta \mid x) \mathrm{d} \theta \\
& -\int_{\forall \theta} \exp [-z \theta] f(\theta \mid x) \mathrm{d} \theta=\exp [a \widehat{\theta}] \int_{\forall \theta} \exp [-\theta(a+z)] f(\theta \mid x) \mathrm{d} \theta-a \widehat{\theta} \int_{\forall \theta} \exp [-z \theta] f(\theta \mid x) \mathrm{d} \theta \\
& +a \int_{\forall \theta} \theta \exp [-z \theta] f(\theta \mid x) \mathrm{d} \theta-\int_{\forall \theta} \exp [-z \theta] f(\theta \mid x) \mathrm{d} \theta=\exp [a \widehat{\theta}] E_{\theta}(\exp [-\theta(z+a) \mid x]) \\
& -a \hat{\theta} E_{\theta}(\exp [-z \theta \mid x])+a E_{\theta}(\theta \exp [-z \theta \mid x])-E(\exp [-z \theta \mid x])=\frac{\partial E_{\theta} L_{w}(\widehat{\theta}, \theta)}{\partial \widehat{\theta}}=a \exp [a \hat{\theta}] E(\exp [-\theta(z+a) \mid x]) \\
& -a E_{\theta}(\exp [-z \theta \mid x])=0 .
\end{aligned}
$$

So, we can find that

$a \exp [a \hat{\theta}] E(\exp [-\theta(z+a) \mid x])-=a E(\exp [-z \theta \mid x])$.

Consequently, the Bayesian estimation of parameter $\theta$ using WLINEX will be

$$
\begin{aligned}
\hat{\theta}_{\mathrm{WBL}} & =\frac{1}{a} \operatorname{Ln} E(\exp [-z \theta])-\frac{1}{a} \operatorname{Ln}(E(\exp [-\theta\{z+a\}])) \\
& =\frac{1}{a} \operatorname{Ln}\left[\frac{E(\exp [-z \theta])}{E(\exp [-\theta(z+a)])}\right] .
\end{aligned}
$$

\section{Bayes Estimation}

In the case of the two-parameter problem, we need to specify a general joint prior for $\alpha$ and $\beta$ which may lead to complicated calculations. Aiming to solve this problem, Soland's method was used. Soland [21] considered a family of joint prior distributions that places continuous distributions on the scale parameter and discrete distributions on the shape parameter.

Suppose that $\alpha$ is restricted to the values $\alpha_{1}, \alpha_{2}, \alpha_{3}, \ldots, \alpha_{k}$ with prior probabilities $\eta_{1}, \eta_{2}, \eta_{3}, \ldots, \eta_{k}$, that is,

$$
P\left(\alpha_{j}\right)=P_{r}\left(\alpha=\alpha_{j}\right)=\eta_{j}, \quad j=1,2,3, \ldots, k,
$$

such that $0<\eta_{j}<1$ and $\sum_{j=1}^{k} \eta_{j}=1$.

Furthermore, suppose that the conditional $\beta$ upon $P_{r}\left(\alpha=\alpha_{j}\right), j=1,2,3, \ldots, k$, has natural conjugate prior as $\left(s_{j}, b_{j}\right)$-gamma with p.d.f

$$
\pi\left(\beta \mid \alpha=\alpha_{j}\right)=\frac{b_{j}^{s_{j}}}{\Gamma\left(s_{j}\right)} \beta^{s_{j}-1} \exp \left[-b_{j} \beta\right], \quad \beta>0, b_{j}, s_{j}>0 .
$$

Combining the likelihood function in (6) and the conditional prior in (20), we get the conditional posterior of $\left(\beta \mid \alpha=\alpha_{j}\right)$ as follows:

$$
\begin{aligned}
\pi^{*}\left(\beta \mid \alpha=\alpha_{j}\right) & =\frac{L(\beta, \alpha ; x) \pi\left(\beta \mid \alpha=\alpha_{j}\right)}{\int_{0}^{\infty} \pi\left(\beta \mid \alpha=\alpha_{j}\right) \mathrm{d} \beta} \\
& =\frac{1}{\Gamma\left(m+s_{j}\right)} v^{m+s_{j}} \beta^{s_{j}-1} \exp \left[-\beta v_{j}\right],
\end{aligned}
$$

where $v_{j}=\left(b_{j}+x_{U(m)}^{\alpha_{j}}\right)$.

In view of the discrete version of Bayes theorem, we obtain the marginal posterior of $\alpha$ as

$$
\begin{aligned}
h\left(\alpha_{j} \mid x\right) & =p_{j}=P_{r}\left(\alpha=\alpha_{j} \mid x\right)=Q \int_{0}^{\infty} \frac{\eta_{j} b_{j}^{s_{j}} \beta^{m+s_{j}-1} \alpha_{j}^{m} u_{j}}{\Gamma\left(s_{j}\right)} \exp \left[-\beta v_{j}\right] \mathrm{d} \beta \\
& =Q \frac{\eta_{j} b_{j}^{s_{j}} \alpha_{j}^{m} u_{j} \Gamma\left(m+s_{j}\right)}{v_{j}^{m+s_{j}} \Gamma\left(s_{j}\right)},
\end{aligned}
$$

where $u_{j}=\prod_{i=1}^{m} x_{U(i)}^{\alpha_{j}-1}$ and $Q$ is a normalized constant given by

$$
Q^{-1}=\sum_{j=1}^{k} \frac{\eta_{j} b_{j}^{s_{j}} \alpha_{j}^{m} u_{j} \Gamma\left(m+s_{j}\right)}{v_{j}^{m+s_{j}} \Gamma\left(s_{j}\right)} .
$$


4.1. Estimates Based on the Squared-Error Loss Function. From (21) and (22), the Bayes estimates of $\beta, \alpha$, and $R(t)$ based on the SEL function are derived, respectively, as

$$
\widehat{\beta}_{\mathrm{BS}}=\int_{0}^{\infty} \sum_{j=1}^{k} p_{j} \beta \pi^{*}\left(\beta \mid \alpha=\alpha_{j}, x\right) \mathrm{d} \beta,
$$

$$
\begin{aligned}
\widehat{\beta}_{\mathrm{BS}} & =\sum_{j=1}^{k} \frac{p_{j}\left(s_{j}+m\right)}{v_{j}}, \\
\widehat{\alpha}_{\mathrm{BS}} & =\sum_{j=1}^{k} p_{j} \alpha_{j}, \\
\widehat{R}_{\mathrm{BS}} & =\int_{0}^{\infty} \sum_{j=1}^{k} p_{j} \exp \left[-\beta t^{\alpha_{j}}\right] \pi^{*}\left(\beta \mid \alpha=\alpha_{j}, x\right) \mathrm{d} \beta=\sum_{j=1}^{k} p_{j}\left[\frac{v_{j}}{v_{j}+t^{\alpha_{j}}}\right]^{s_{j}+m} .
\end{aligned}
$$

4.2. Estimates Based on the LINEX Error Loss Function. Under the LINEX loss function (12), the Bayes estimate of a function $\gamma(\alpha, \beta)$ is given by (13).

The Bayes estimator for the scale parameter $\beta$ is given by

$$
\begin{aligned}
\widehat{\beta}_{\mathrm{BL}} & =-\frac{1}{a} \operatorname{Ln}\left[\int_{0}^{\infty} \sum_{j=1}^{k} p_{j} \exp [-a \beta] \pi^{*}\left(\beta \mid \alpha=\alpha_{j}, x\right) \mathrm{d} \beta\right] \\
& =-\frac{1}{a} \operatorname{Ln}\left[\sum_{j=1}^{k} p_{j}\left(1+\frac{a}{v_{j}}\right)\right]^{-\left(S_{J}+m\right)},
\end{aligned}
$$

and the Bayes estimator for $\alpha$ is given by

$$
\widehat{\alpha}_{\mathrm{BL}}=-\frac{1}{a} \operatorname{Ln}\left[\sum_{j=1}^{k} p_{j} \exp \left[-a \alpha_{j}\right]\right]
$$

Similarly, the Bayes estimator for the reliability function $R(t)$ is given by which, upon using (20), simplifies to

$$
\widehat{R}(t)_{\mathrm{BL}}=-\frac{1}{a} \operatorname{Ln}\left[\int_{0}^{\infty} \sum_{j=1}^{k} p_{j} \exp [-a R(t)] \pi^{*}\left(\beta \mid \alpha=\alpha_{j}, x\right) \mathrm{d} \beta\right],
$$

where $R(t)$ is given in (3). By using the exponential series, after some simplifications, we obtain

$$
\widehat{R}(t)_{\mathrm{BL}}=-\frac{1}{a} \operatorname{Ln}\left[\sum_{j=1}^{k} \sum_{i=0}^{\infty} \frac{(-a)^{i}}{i !}\left(1+\frac{i t^{\alpha_{j}}}{v_{j}}\right)^{-\left(m+s_{j}\right)}\right] .
$$

4.3. Estimates Based on the Weighted LINEX Loss Function. Under the weighted LINEX loss function (14), the Bayes estimate of a function $\gamma(\alpha, \beta)$ is given by (18).

The Bayes estimator for the scale parameter $\beta$ is given by

$$
\widehat{\beta}_{\mathrm{WBL}}=\frac{1}{a} \operatorname{Ln}\left[\frac{E(\exp \{-z \beta\})}{E(\exp \{-\beta(z+a)\})}\right]=\frac{1}{a} \operatorname{Ln}\left[\frac{I_{1}}{I_{2}}\right] \text {, }
$$

where

$$
\begin{aligned}
& I_{1}=\left[\int_{0}^{\infty} \sum_{j=1}^{m} p_{j} \exp [-z \beta] \pi^{*}\left(\beta \mid \alpha=\alpha_{j}, x\right) \mathrm{d} \beta\right]=\left[\sum_{j=1}^{m} p_{j}\left(1+\frac{z}{v_{j}}\right)\right]^{-\left(S_{J}+m\right)}, \\
& I_{2}=\left[\int_{0}^{\infty} \sum_{j=1}^{m} p_{j} \exp [-(a+z) \beta] \pi^{*}\left(\beta \mid \alpha=\alpha_{j}, x\right) \mathrm{d} \beta\right]=\left[\sum_{j=1}^{m} p_{j}\left(1+\frac{z+a}{v_{j}}\right)\right]^{-\left(S_{J}+m\right)}, \\
& \widehat{\alpha}_{\mathrm{WBL}}=\frac{1}{a} \operatorname{Ln}\left[\frac{E(\exp \{-z \alpha\})}{E(\exp \{-\alpha(z+a)\})}\right]=\frac{1}{a} \operatorname{Ln}\left[\frac{I_{3}}{I_{4}}\right],
\end{aligned}
$$

and the Bayes estimator for $\alpha$ is given by 
where

$$
\begin{aligned}
& I_{3}=\sum_{j=1}^{k} p_{j} \exp \left[-z \alpha_{j}\right], \\
& I_{4}=\sum_{j=1}^{k} p_{j} \exp \left[-(a+z) \alpha_{j}\right] .
\end{aligned}
$$

Similarly, the Bayes estimator $\widehat{R}_{\mathrm{WBL}}(t)$ of the reliability function $R(t)$ is given by

$$
\begin{aligned}
\widehat{R}(t)_{\mathrm{BL}} & =-\frac{1}{a} \operatorname{Ln}\left[\int_{0}^{\infty} \sum_{j=1}^{k} p_{j} \exp [-a R(t)] \pi^{*}\left(\beta \mid \alpha=\alpha_{j}, x\right) \mathrm{d} \beta\right], \\
\widehat{R}_{\mathrm{WBL}}(t) & =\frac{1}{a} \operatorname{Ln}\left[\frac{E \exp [-z R(t)]}{E(\exp [-(a+z) R(t)])}\right]=\frac{1}{a} \operatorname{Ln}\left[\frac{I_{5}}{I_{6}}\right],
\end{aligned}
$$

where

$$
\begin{aligned}
& I_{5}=\sum_{j=1}^{k} \sum_{i=0}^{\infty} \frac{(-z)^{i}}{i !}\left(1+\frac{i t^{\alpha_{j}}}{v_{j}}\right)^{-\left(m+s_{j}\right)}, \\
& I_{6}=\sum_{j=1}^{k} \sum_{i=0}^{\infty} \frac{(-(z+a))^{i}}{i !}\left(1+\frac{i t^{\alpha_{j}}}{v_{j}}\right)^{-\left(m+s_{j}\right)} .
\end{aligned}
$$

To begin with the calculation, we have to choose the values of $\left(\alpha_{j}, \eta_{j}\right)$ and the hyperparameters $\left(s_{j}, b_{j}\right)$ in the conjugate prior (20), for $j=1,2, \ldots, k$. The values of the previous pairs are quite easy to specify, but to determine $\left(s_{j}, b_{j}\right)$, there is a need to condition prior ideas about $\beta$ on each $\alpha_{j}$, in turn, which is not easy in implementation. An alternative method that is based on the expected value of the reliability function $R(t)$ conditional on $\alpha=\alpha_{j}$ to obtain the values $\left(s_{j}, b_{j}\right)$ is given using (20) by

$$
E_{\beta \mid \alpha_{j}}\left[R(t) \mid \alpha=\alpha_{j}=\frac{b_{j}^{s_{j}} \beta^{s_{j}-1}}{\Gamma\left(s_{j}\right)} \exp \left[-b_{j} \beta\right] \mathrm{d} \alpha=\left(1+\frac{t^{\alpha_{j}}}{b_{j}}\right)^{-s_{j}} .\right.
$$

So, suppose now that previous ideas about the lifetime distribution enable us to specify two values including $\left(R\left(t_{1}\right), t_{1}\right)$ and $\left(R\left(t_{2}\right), t_{2}\right)$. Therefore, for these prior values $R\left(t=t_{1}\right)$ and $R\left(t=t_{2}\right)$, the values of $s_{j}$ and $b_{j}$, for each value of $\alpha_{j}$, can be computed numerically from (36). In the case of nonexistence of previous ideas, one may use a nonparametric procedure for estimating the corresponding different values of $R(t)$ as in Martz and Waller [22].

\section{Application with Real Data}

To demonstrate the techniques of estimation which are developed in this work, we study the real dataset which was used before by Lawless [23]. These data were taken from the study by Nelson [24]. These data record the time to breakdown of an insulating fluid between electrodes at a voltage of $34 \mathrm{kV}$ (minutes). The 19 times (complete sample) to breakdown are $0.96,4.15,0.19,0.78,8.01,31.75,7.35,6.50$, $8.27,33.91,32.52,3.16,4.85,2.78,4.67,1.31,12.06,36.71$, and 72.89 .

Note: Lawless (1982) applied the ML method on these data to estimate the parameters $\alpha, \beta$, and $R(t)$, where $\widetilde{\beta}=0.145, \widetilde{\alpha}=0.771$, and $\widetilde{R}(t)=0.606$ at $t=5$ and $z=3$.

Thus, the seven upper record values clearly are $0.96,4.15$, 8.01, 31.75, 33.91, 36.71, and 72.89.

A Weibull distribution represents the time to breakdown for a fixed voltage level as it was mentioned by engineering considerations. The calculations for this application are conducted by using the gamma prior for the scale parameter, while the discrete distribution is applied for the shape parameter. The hyperparameters of the gamma prior (36) and the values of $\alpha_{j}$ are derived by the following steps:

(1) Depending on the previous seven upper records, we use a nonparametric procedure $R\left(t_{i}=X_{U(i)}\right)=($ $(m-i+0.625) /(m+0.25)), \quad i=1,2,3, \ldots, m$, with $m=7$ to estimate two values of the reliability function. Therefore, the reliability function for times $t_{1}=8.01$ and $t_{2}=33.91$ is $R\left(t_{1}\right)=0.638$ and $R\left(t_{2}\right)=0.362$, respectively.

(2) Assuming that $\alpha_{j} 0.5,0.55,0.6, \ldots, 0.95$, the value of the MLE of parameter $\alpha$ from (8) is computed $\left(\widehat{\alpha}_{\mathrm{Ml}}=0.599\right)$.

(3) The two prior values obtained in Step 1 are substituted into (34), where $s_{j}$ and $b_{j}$ are solved numerically for each given $\alpha_{j}, j=1,2,3, \ldots, 10$, using the Newton-Raphson method.

Table 1 shows the values of the hyperparameters and the posterior probabilities derived for each value in $\alpha_{j}$, while Table 2 displays the computed estimations of $\alpha, \beta$, and $R(t)$ using ML estimates $(.)_{\mathrm{ML}}$ and the Bayes estimates $\left((.)_{\mathrm{BS}}\right.$, $(.)_{\mathrm{BL}}$, and $\left.(.)_{\mathrm{WBL}}\right)$.

According to the smallest differences $|\widehat{\beta}-\widetilde{\beta}|$ and $|\widehat{\alpha}-\widetilde{\alpha}|$, the developed method in this work (WLINEX) has the best performance in estimating the scale parameter $\beta$ and shape parameter $\alpha$ as it is shown in Table 2 . With respect to the reliability function $R(t)$, the developed method is better than 
TABLE 1: Prior information, hyperparameters, and posterior probabilities.

\begin{tabular}{|c|c|c|c|c|c|c|}
\hline$j$ & $\alpha_{j}$ & $\eta_{j}$ & $s_{j}$ & $b_{j}$ & $u_{j}$ & $p_{j}$ \\
\hline 1 & 0.5 & 0.1 & 300604 & $1.66018^{*} 10^{6}$ & 0.000104 & 0.005087 \\
\hline 2 & 0.55 & 0.1 & $1.33736^{*} 10^{6}$ & $9.01977^{*} 10^{6}$ & 0.000261 & 0.005984 \\
\hline 3 & 0.60 & 0.1 & 5.73266 & 42.7212 & 0.000653 & 0.034157 \\
\hline 4 & 0.65 & 0.1 & 2.42594 & 18.9945 & 0.001632 & 0.097530 \\
\hline 5 & 0.70 & 0.1 & 1.56698 & 12.9143 & 0.004083 & 0.149181 \\
\hline 6 & 0.75 & 0.1 & 1.17088 & 10.1731 & 0.010213 & 0.171266 \\
\hline 7 & 0.80 & 0.1 & 0.942272 & 8.6422 & 0.025545 & 0.167616 \\
\hline 8 & 0.85 & 0.1 & 0.793059 & 7.68699 & 0.063897 & 0.148551 \\
\hline 9 & 0.90 & 0.1 & 0.687747 & 7.05194 & 0.159828 & 0.123184 \\
\hline 10 & 0.95 & 0.1 & 0.609272 & 6.61438 & 0.399785 & 0.097443 \\
\hline
\end{tabular}

TABle 2: Estimates of $\alpha, \beta$, and $R(t)$ when $t=5$ and $z=3$.

\begin{tabular}{|c|c|c|c|c|c|c|}
\hline Method & $\widehat{\alpha}$ & $|\widehat{\alpha}-\tilde{\alpha}|$ & $\widehat{\beta}$ & $|\widehat{\beta}-\widetilde{\beta}|$ & $\widehat{R}(t)$ & $|\widehat{R}(t)-\widetilde{R}(t)|$ \\
\hline$(.)_{\mathrm{ML}}$ & 0.599 & 0.172 & 0.536 & 0.391 & 0.245 & 0.361 \\
\hline$(.)_{\mathrm{BS}}$ & 0.786 & 0.015 & 0.202 & 0.057 & 0.514 & 0.092 \\
\hline$(.)_{\mathrm{BL}}(a=-1)$ & 0.791 & 0.020 & 0.206 & 0.061 & 0.521 & 0.085 \\
\hline (.) $)_{\mathrm{BL}}(a=-2)$ & 0.796 & 0.025 & 0.210 & 0.065 & 0.529 & 0.077 \\
\hline$(.)_{\mathrm{BL}}(a=2)$ & 0.776 & 0.005 & 0.195 & 0.050 & 0.498 & 0.108 \\
\hline (.) $)_{\mathrm{WBL}}(a=-1)$ & 0.761 & 0.010 & 0.185 & 0.040 & 0.475 & 0.131 \\
\hline (.) $)_{\mathrm{WBL}}(a=-2)$ & 0.766 & 0.005 & 0.188 & 0.043 & 0.483 & 0.123 \\
\hline (.) $)_{\mathrm{WBL}}(a=2)$ & 0.746 & 0.025 & 0.176 & 0.031 & 0.452 & 0.154 \\
\hline
\end{tabular}

the ML method and comes in the third level of accuracy after BL and BS methods with small differences. Finally, these results reveal that the proposed model in this work is promising and can be applied in other real environments.

\section{Data Availability}

The data were taken from the study by Nelson [24]. These data record the time to breakdown of an insulating fluid between electrodes at a voltage of $34 \mathrm{kV}$ (minutes).

\section{Conflicts of Interest}

The author declares that there are no conflicts of interest.

\section{References}

[1] H. N. Nagaraja, "Record values and related statistics - a review," Communications in Statistics - Theory and Methods, vol. 17, no. 7, pp. 2223-2238, 1988.

[2] M. Ahsanullah, Introduction to Record Statistics, NOVA Science Publishers Inc., New York, NY, USA, 1995.

[3] B. C. Arnold, N. Balakrishnan, and H. N. Nagaraja, Records, John Wiley \& Sons, New York, NY, USA, 1998.

[4] S. Baratpour, J. Ahmadi, and N. R. Arghami, "Entropy properties of record statistics," Statistical Papers, vol. 48, no. 2, pp. 197-213, 2007.

[5] A. H. Khan and I. A. Shah, "Distributional properties of order statistics and record statistics," Pakistan Journal of Statistics and Operation Research, vol. 8, no. 3, pp. 573-581, 2012.

[6] A. S. Hassan, M. Abd-Allah, and H. F. Nagy, "Bayesian analysis of record statistics based on generalized inverted exponential model," International Journal on Advanced Science, Engineering and Information Technology, vol. 8, no. 2, pp. 323-335, 2018.
[7] N. Balakrishnan and P. Chan, "Record values from rayleigh and weibull distributions and associated inference," National Institute of Standards and Technology Journal of Research, vol. 866, pp. 41-51, 1993.

[8] S. Omid, R. Azimi, and M. Babanezhad, "Empirical Bayes estimators of parameter and reliability function for compound Rayleigh distribution under record data," American Journal of Theoretical and Applied Statistics, vol. 1, no. 1, pp. 12-15, 2012.

[9] A. Ahmed, A. H. Soliman, A. H. Abd Ellah, and K. S. Sultan, "Comparison of estimates using record statistics from Weibull model: Bayesian and non-Bayesian approaches," Computational Statistics \& Data Analysis, vol. 51, pp. 2065-2077, 2006.

[10] K. S. Sultan, "Bayesian estimates based on record values from the Inverse Weibull lifetime model," Quality Technology \& Quantitative Management, vol. 5, no. 4, pp. 363-374, 2008.

[11] F. S. Al-Duais, "Bayesian analysis of record statistic from the Inverse Weibull distribution under balanced loss function," Mathematical Problems in Engineering, vol. 2021, Article ID 6648462, 9 pages, 2021.

[12] A. Asgharzadeh and A. Fallah, "Estimation and prediction for exponentiated family of distributions based on records," Communications in Statistics-Theory and Methods, vol. 40, pp. 68-83, 2011.

[13] S. N. Sindi, G. R. Al-Dayian, and S. H. Shahbaz, "Inference for exponentiated general class of distributions based on record values," Pakistan Journal of Statistics and Operation Research, vol. 13, no. 3, pp. 575-587, 2017.

[14] K. S. Sultan, M. E. Moshref, and N. S. Abd-El-Hakim, "Estimation of parameters of Lomax distribution based on record values," The Egyptian Statistical Journal, vol. 45, no. 2, pp. 135-142, 2001.

[15] F. S. Al-Duais and M. Y. Hmood, "Bayesian and non-Bayesian estimation of the Lomax model based on upper record values under weighted LINEX loss function," Periodicals of Engineering and Natural Sciences (PEN), vol. 8, no. 3, pp. 17861794, 2020, p. 
[16] A. Pak and S. Dey, "Statistical inference for the power Lindley model based on record values and inter-record times," Journal of Computational and Applied Mathematics, vol. 347, pp. 156-172, 2019, p.

[17] A. M. Abd-Elrahman and M. A. Mohamad, "Estimation of mixed exponentiated Weibull parameters in life testing," International Mathematical Forum, vol. 11, no. 16, pp. 769781, 2016.

[18] A. M. Abd-Elrahman and M. A. Mohammed, "Estimation of a nonlinear discriminant function from a mixture of two exponentiated-Weibull distributions," Journal of Mathematics and Computer Science, vol. 6, no. 5, pp. 907-921, 2016, p.

[19] A. Asgharzadeh, R. Valiollahi, A. Fallah, and S. Nadarajah, "Bayesian inference and prediction for normal distribution based on records," Statistica, vol. 78, no. 1, pp. 15-36, 2018.

[20] J. Ahmadi, M. Doostparast, and A. Parsian, "Estimation and prediction in a two-parameter exponential distribution based on k-record values under LINEX loss function," Communications in Statistics - Theory and Methods, vol. 34, no. 4, pp. $795-805,2005$, p.

[21] R. M. Soland, "Bayesian analysis of the Weibull Process with unknown scale and shape parameters," IEEE Transactions on Reliability, vol. 18, no. 4, pp. 181-184, 1969.

[22] Hy.F. Martz and R. A. Waller, Bayesian Reliability Analysis, Wiley, New York, NY, USA, 1982.

[23] J. F. Lawless, Statistical Model \& Methods for Lifetime Data, Wiley, New York, NY, USA, 1982.

[24] W. B. Nelson, Applied Life Data Analysis, John Wiley \& Sons, New York, NY, USA, 1982. 